\title{
THE COLLOCATION METHOD FOR FIRST-KIND BOUNDARY INTEGRAL EQUATIONS ON POLYGONAL REGIONS
}

\author{
YI YAN
}

\begin{abstract}
In this paper the collocation method for first-kind boundary integral equations, by using piecewise constant trial functions with uniform mesh, is shown to be equivalent to a projection method for second-kind Fredholm equations. In a certain sense this projection is an interpolation projection. By introducing this technique of analysis, we particularly consider the case of polygonal boundaries. We give asymptotic error estimates in $L_{2}$ norm on the boundaries, and some superconvergence results for the single layer potential.
\end{abstract}

\section{INTRODUCTION}

The collocation method is a frequently used numerical technique in practical engineering problems because of its easy implementation, particularly for boundary integral equations for two-dimensional boundary value problems. One of the applications is to boundary integral equations of the first kind with logarithmic kernel (Symm's integral equation) on a closed boundary $\Gamma$,

$$
-\int_{\Gamma} \log |x-y| g(y) d y=f(x), \quad x \in \Gamma \subset R^{2},
$$

where $d y$ denotes the element of arc length at a point $y \in \Gamma$. For the collocation method for this equation, the trial space is usually chosen as a $B$-spline space, and the collocation points are chosen appropriately according to the degree of the spline.

The asymptotic behavior of the collocation solution of boundary integral equations on a smooth boundary has been analyzed theoretically and experimentally by many authors. Particularly, the recent results of Arnold, Wendland, Saranen and Schmidt in $[2,3,13,15,16,14]$ cover the first-kind boundary integral equation of potential problems. In their work, basically two general techniques of analysis have been introduced. One is based on an equivalence of some collocation methods with a certain Petrov-Galerkin method, and the other is based on simple Fourier analysis. Both of these two approaches rely on the strong ellipticity of the boundary integral operator. However, the theoretical analysis of the collocation method has not yet been sufficiently developed

Received June 6, 1988; revised April 11, 1989.

1980 Mathematics Subject (lassification (1985 Revision). Primary 65N35, 65R20; Secondary $45 \mathrm{~L} 10$.

Key words and phrases. Collocation method, first-kind boundary integral equations, polygonal regions. 
to cover the case of a polygonal boundary $\Gamma$, which occurs more frequently in practical engineering problems. The reason is that in such cases the integral operator is no longer a pseudodifferential operator with a logarithmic principal part, so that the two techniques of analysis in the papers cited above are not directly applicable. There are only a few works which analyze the collocation method on a polygonal boundary $\Gamma[8,9]$. In these, Costabel and Stephan introduce local Mellin transforms and a weighted norm, and obtain error estimates for collocation with piecewise linear trial functions.

In the present paper, we introduce a different approach to the analysis of convergence of midpoint collocation with piecewise constant trial functions, and give asymptotic error estimates in the $L_{2}$ norm on the boundary, and some superconvergence results for the single layer potential.

The approach is to view the collocation method for the first-kind integral equation as equivalent to a projection method for a second-kind Fredholm equation with a particular projection operator. This particular projection is derived from the collocation method for equation (1) on a circle. In $\S 2$ we present in detail an analysis of the projection operator by employing the spectral method. Although this analysis here is only for the piecewise constant function space, we believe that similar results are possible for spline function spaces of arbitrary degree if the mesh is uniform. Thus, this approach may be available for any spline function spaces with uniform mesh.

Let $\Gamma$ be a polygon with corner points $v_{j}, j=1, \ldots, \gamma$. On the polygon $\Gamma$ let $\Delta_{n}=\left\{z_{0}, \ldots, z_{n-1}\right\} \subset \Gamma$ be an equally spaced mesh, and $\bar{\Delta}_{n}$ be the corresponding midpoint mesh. Then we adopt $\Delta_{n}$ as the break points of trial functions, and $\bar{\Delta}_{n}$ as the collocation points. The trial function space is a piecewise constant space $S^{h}(\Gamma)$ with break points $\Delta_{n}$, where $h=2 \pi / n$. The collocation method approximates the solution $g$ by $g^{h} \in S^{h}(\Gamma)$, such that

$$
-\int_{\Gamma} \log |x-y| g^{h}(y) d y=f(x) \text { for } x \in \bar{\Delta}_{n} \text {. }
$$

For convenience of our analysis, we represent equations (1) and (2) as follows. We parametrize the polygon $\Gamma$ by a $2 \pi$-periodic function $v(s): \mathbf{R} / 2 \pi \mathbf{Z} \rightarrow \Gamma$, with the parameter $s$ proportional to the arc length; that is, $\left|v^{\prime}(s)\right| \equiv d(\Gamma) / 2 \pi$, where $d(\Gamma)$ is the length of $\Gamma$. Suppose that the corner points are at $v_{1}=$ $v\left(s_{1}\right), \ldots, v_{\gamma}=v\left(s_{\gamma}\right)$, with $-\pi=s_{0}<s_{1}<\cdots<s_{\gamma}<s_{\gamma+1}=\pi, v_{0}=v\left(s_{0}\right)=$ $v_{i+1}=v\left(s_{i+1}\right)$. Then $v(s)$ can be described as

$$
v(s)=v_{j}+\frac{s-s_{j}}{s_{j+1}-s_{j}}\left(v_{j+1}-v_{j}\right) \text { for } s_{j} \leq s \leq s_{j+1}, j=0, \ldots, \gamma .
$$

Thus, a transformed form of equation ( 1 ) is obtained,

$$
-\frac{1}{\pi} \int_{-\pi}^{\pi} \log |v(s)-v(\sigma)| w(\sigma) d \sigma=\bar{f}(s), \quad s \in[-\pi, \pi],
$$

with $w(s)=\left|v^{\prime}(s)\right| g(v(s))$ and $\bar{f}(s)=\frac{1}{\pi} f(v(s))$. This equation can also be written in the form of an operator equation
$(1)^{*}$
$K w=\bar{f}$. 
Correspondingly, we have a transformed collocation equation. Let $\sigma_{j}=$ $-\pi+h j$ for $j=0,1, \ldots, n$, and $\sigma_{j+1 / 2}=\sigma_{j}+\frac{1}{2} h$ for $j=0,1, \ldots, n-1$. Further assume that the function $v(s)$ satisfies $z_{j}=v\left(\sigma_{j}\right), j=0, \ldots, n-1$. Set $\Pi=\left\{\sigma_{j}: j=0,1, \ldots, n\right\}$, and let $S^{h}(\Pi)$ be a piecewise constant function space on $[-\pi, \pi]$ with break points $\Pi$. Suppose that $Q^{h}$ is an interpolation projection from $C[-\pi, \pi]$ to $S^{h}(\Pi)$ defined by

$$
Q^{h} v(s)=\sum_{j=0}^{n-1} v\left(\sigma_{j+1 / 2}\right) X_{j}(s),
$$

where

$$
X_{j}(s)= \begin{cases}1, & s \in\left(\sigma_{j}, \sigma_{j+1}\right), \\ 0, & \text { otherwise } .\end{cases}
$$

Then the transformed form of the collocation equation (2), in operator form, is

$$
Q^{h} K w^{h}=Q^{h} \bar{f}
$$

with $w^{h} \in S^{h}(\Pi)$.

What we treat here is the collocation method based on the piecewise constant trial function space $S^{h}(\Gamma)$ with an equally spaced mesh on the whole polygon $\Gamma$; or, in other words, with a uniform mesh. The corner points $v_{j}$, $j=1, \ldots, \gamma$, then may not belong to the uniform mesh $\Delta_{n}$. In practical computation one often chooses the corner points as a part of the mesh points to obtain an easier integration around the corners. In this sense the treatment here is not completely satisfactory. However, the corner points $v_{j}, j=1, \ldots, \gamma$, can be put into a uniform mesh $\Delta_{h}$ without difficulty for many practical cases such as squares, or polygons of equal sides. We also know that the singularities of the solution $g(v(s))$ at corners often degrade the rate of convergence in practical computation (see [17, 18]). But as shown in [19] for the Galerkin method, the mesh grading method can be exploited to restore the rate of convergence. Hence, we can hope to adopt the mesh grading technique to restore the rate of convergence for the collocation method as well. However, up to now, its theory has not been developed.

In order to give a more precise analysis, we now introduce some notation. We shall consider equation (1) and its collocation approximation $(2)^{*}$ in a family of $2 \pi$-periodic Sobolev spaces. Each $2 \pi$-periodic function $v$ has a Fourier expansion

$$
v(s)=\frac{1}{\sqrt{2 \pi}} \sum_{m \in \mathbf{Z}} \hat{v}(m) e^{i m s},
$$

where the Fourier coefficients are given by the formula

$$
\hat{v}(m)=\frac{1}{\sqrt{2 \pi}} \int_{-\pi}^{\pi} v(s) e^{-i m s} d s .
$$


Let $\mathbf{Z}^{*}=\mathbf{Z} \backslash\{0\}$. For $t \in \mathbf{R}$ define the inner product

$$
(u, v)_{t}=\hat{u}(0) \overline{\hat{v}(0)}+\sum_{m \in \mathbf{Z}^{*}}|m|^{2 t} \hat{u}(m) \overline{\hat{v}(m)} .
$$

The Sobolev space $H^{t}(2 \pi)$ consists of all $2 \pi$-periodic and complex functions $v$ for which the norm $|u|_{t}=\sqrt{(u, u)_{t}}$ is finite. A useful fact is that $H^{0}=L_{2}(2 \pi)$, and $(u, v)_{0}=\int_{-\pi}^{\pi} u(\sigma) \bar{v}(\sigma) d \sigma=(u, v)$, which implies that the $L_{2}(2 \pi)$ norm is just the $H^{0}$ norm. Below we shall adopt the notation $|\cdot|_{0}$ for the $L_{2}$ norm without any remark.

\section{Collocation on a CIRCLE}

Since the simplest case of the first-kind integral equation (1) is for a circle, a detailed analysis of its collocation method is carried out in this section by employing the spectral analysis technique. Earlier convergence results have been obtained in $[3,13,14]$ by use of the Fourier analysis method.

In the case of a circular contour, equation (1) ${ }^{*}$ may be written as (see [20]),

$$
A w=\bar{f},
$$

where $A w(s)=\int_{-\pi}^{\pi} \Lambda(s-\sigma) w(\sigma) d \sigma$ with

$$
\Lambda(s-\sigma)=-\frac{1}{\pi} \log \left|2 e^{-1 / 2} \sin \frac{s-\sigma}{2}\right| .
$$

The collocation solution $w_{h}$ satisfies

$$
Q^{h} A w_{h}=Q^{h \bar{f}}=Q^{h} A w .
$$

Letting $A_{h}$ denote the restriction of $A$ on $S^{h}(\Pi), Q^{h} A_{h}$ is invertible on $S^{h}(\Pi)$ from Theorem 1 below. Let $B^{h}=\left(Q^{h} A_{h}\right)^{-1} Q^{h} A$. Then $w_{h}$ can be represented as

$$
w_{h}=B^{h} w \text {. }
$$

In the following we shall show that $B^{h}$ is a bounded operator. For this purpose we consider a complex piecewise constant function space $\bar{S}^{h}(\Pi)=\left\{v_{1}+\right.$ $\left.i v_{2}: v_{1}, v_{2} \in S^{h}(\Pi)\right\}$, and introduce the following basis of the space $\bar{S}^{h}(\Pi)$. We assume for convenience that $n$ is an odd number throughout this section. Let us define $\Lambda_{h}=\{p \in \mathbf{Z}:|p| \leq(n-1) / 2\}, \Lambda_{h}^{*}=\Lambda_{h} \backslash\{0\}$. Then orthogonal basis functions for $\bar{S}^{h}(\Pi)$ are defined by

$$
e_{h}^{p}(s)=\sum_{j=0}^{n-1} e^{i h j p} X_{j}(s), \quad p \in \Lambda_{h},
$$

with $\left(e_{h}^{p}, e_{h}^{p^{\prime}}\right)=2 \pi \delta_{p, p^{\prime}}$ for $p, p^{\prime} \in \Lambda_{h}$. The definition of the functions $e_{h}^{p}(s)$, when they appear later, is extended to $p \in \mathbf{Z}$ without any remark. One of the 
advantages of introducing the basis functions $e_{h}^{p}(s)$ is that for any $f_{h} \in S^{h}(\Pi)$, in terms of its corresponding discrete Fourier coefficients

$$
F^{h} f_{h}(p)=\frac{1}{\sqrt{2 \pi}} \int_{-\pi}^{\pi} f_{h}(\sigma) e_{h}^{-p}(\sigma) d \sigma, \quad p \in \Lambda_{h}
$$

the function $f_{h}$ can be represented as

$$
f_{h}(s)=\frac{1}{\sqrt{2 \pi}} \sum_{p \in \Lambda_{h}} F^{h} f_{h}(p) e_{h}^{p}(s)
$$

This representation looks like the discrete Fourier expansion of the function $f_{h}(s)$. Another advantage is that the basis functions $e_{h}^{p}(s)$ are the eigenfunctions of the collocation operator $Q^{h} A_{h}$.

Theorem 1. $Q^{h} A_{h}$ in $\bar{S}^{h}(\Pi)$ has the eigenvalues $\left\{\lambda_{p}: p \in \Lambda_{h}\right\}$ given by

(4) $\lambda_{p}=\left\{\begin{array}{l}1, \quad p=0, \\ \frac{n}{\pi} \sin \frac{\pi|p|}{n} \sum_{k=0}^{\infty}(-1)^{k}\left(\frac{1}{(k n+|p|)^{2}}+\frac{1}{(k n+n-|p|)^{2}}\right), \quad p \in \Lambda_{h}^{*},\end{array}\right.$

and the corresponding eigenfunctions are $e_{h}^{p}(s)$.

This theorem implies that $\lambda_{0}$ is a simple eigenvalue, whereas for $p \in \Lambda_{h}^{*}$ the eigenvalues $\lambda_{p}$ and $\lambda_{-p}$ are degenerate, so that there are two independent eigenfunctions. (If $n$ were an even integer, the expression for the eigenvalues would remain the same, but there would then be second simple eigenvalue, namely $\lambda_{n / 2}$. )

Theorem 1 also implies that $Q^{h} A_{h}$ is invertible on $S^{h}(\Pi)$, because all the eigenvalues $\lambda_{p}$ are greater than 0 .

The eigenvalues $\lambda_{p}$ have been discussed in the work of F. R. de Hoog [10] and S. Christiansen [7]. However, the eigenvalues $\lambda_{p}$ given in (4) are more explicit.

Proof of Theorem 1. If $Q^{h} A_{h} e_{h}^{p}(s)=\lambda_{p} e_{h}^{p}(s)$ with $\lambda_{p}$ real, by conjugating we obtain $Q^{h} A_{h} e_{h}^{-p}(s)=\lambda_{p} e_{h}^{-p}(s)=\lambda_{-p} e_{h}^{-p}(s)$. Thus we only need a proof for $p \geq 0$. As in [20],

$$
A w(s)=\frac{1}{\sqrt{2 \pi}}\left(\sum_{m \in \mathbf{Z}^{*}}|m|^{-1} \hat{w}(m) e^{i m s}+\hat{w}(0)\right)
$$


By a simple calculation,

$$
\begin{aligned}
A X_{j}\left(\sigma_{r+1 / 2}\right) & =\frac{1}{2 \pi}\left(\sum_{m \in \mathbf{Z}^{*}}|m|^{-1} \int_{\sigma_{j}}^{\sigma_{j+1}} e^{-i m \sigma} d \sigma e^{i m \sigma_{r+1 / 2}}+h\right) \\
& =\frac{1}{2 \pi}\left(2 \sum_{m=1}^{\infty} m^{-1} \operatorname{Re}\left(\int_{\sigma_{j}}^{\sigma_{j+1}} e^{-i m \sigma} d \sigma e^{i m \sigma_{r+1 / 2}}\right)+h\right) \\
& =\frac{1}{2 \pi}\left(2 \sum_{m=1}^{\infty} m^{-1} \operatorname{Re}\left(\frac{2 \sin (m h / 2)}{m} e^{i m(r-j) h}\right)+h\right) \\
& =\frac{1}{2 \pi}\left(2 \sum_{k=0}^{\infty} \sum_{l=1}^{n} \frac{2 \sin ((k n+l) h / 2)}{(k n+l)^{2}} \operatorname{Re}\left(e^{i(k n+l)(r-j) h}\right)+h\right) \\
& =\frac{1}{2 \pi}\left(2 \sum_{k=0}^{\infty} \sum_{l=1}^{n} \frac{2(-1)^{k} \sin (l h / 2)}{(k n+l)^{2}} \operatorname{Re}\left(e^{i l(r-j) h}\right)+h\right) \\
& =\frac{1}{2 \pi}\left(\sum_{l=1}^{n-1} 4 \sin \frac{l h}{2} \operatorname{Re}\left(e^{i l(r-j) h}\right) \sum_{k=0}^{\infty} \frac{(-1)^{k}}{(k n+l)^{2}}+h\right) .
\end{aligned}
$$

Thus,

$$
\begin{aligned}
& A e_{h}^{p}\left(\sigma_{r+1 / 2}\right) \\
& \quad=\frac{1}{2 \pi}\left(\sum_{l=1}^{n-1} 2 \sin \frac{l h}{2} \sum_{j=0}^{n-1} e^{i p j h} 2 \operatorname{Re}\left(e^{i l(r-j) h}\right) \sum_{k=0}^{\infty} \frac{(-1)^{k}}{(k n+l)^{2}}+h \sum_{j=0}^{n-1} e^{i p j h}\right) .
\end{aligned}
$$

However, for $p+l \geq 0$,

$$
\sum_{j=0}^{n-1} e^{i p j h} 2 \operatorname{Re}\left(e^{i l(r-j) h}\right)= \begin{cases}n e^{i p r h}, & l=p \text { or } l=n-p \\ 0, & \text { otherwise }\end{cases}
$$

It follows that

$$
A e_{h}^{p}\left(\sigma_{r+1 / 2}\right)=\left\{\begin{array}{r}
1, \quad p=0, \\
\frac{n}{\pi} \sin \frac{\pi p}{n} \sum_{k=0}^{\infty}(-1)^{k}\left(\frac{1}{(k n+p)^{2}}+\frac{1}{(k n+n-p)^{2}}\right) e_{h}^{p}\left(\sigma_{r+1 / 2}\right), \\
1 \leq p \leq \frac{n-1}{2} .
\end{array}\right.
$$

The proof is completed.

From (5) we find that $A X_{j}\left(\sigma_{r+1 / 2}\right)=A X_{r}\left(\sigma_{j+1 / 2}\right)$. This implies a useful symmetry property: for $\phi, \psi \in \bar{S}^{h}(\Pi)$,

$$
\left(Q^{h} A_{h} \phi, \psi\right)=\left(\phi, Q^{h} A_{h} \psi\right),
$$

and therefore

$$
\left(\left(Q^{h} A_{h}\right)^{-1} \phi, \psi\right)=\left(\phi,\left(Q^{h} A_{h}\right)^{-1} \psi\right)
$$




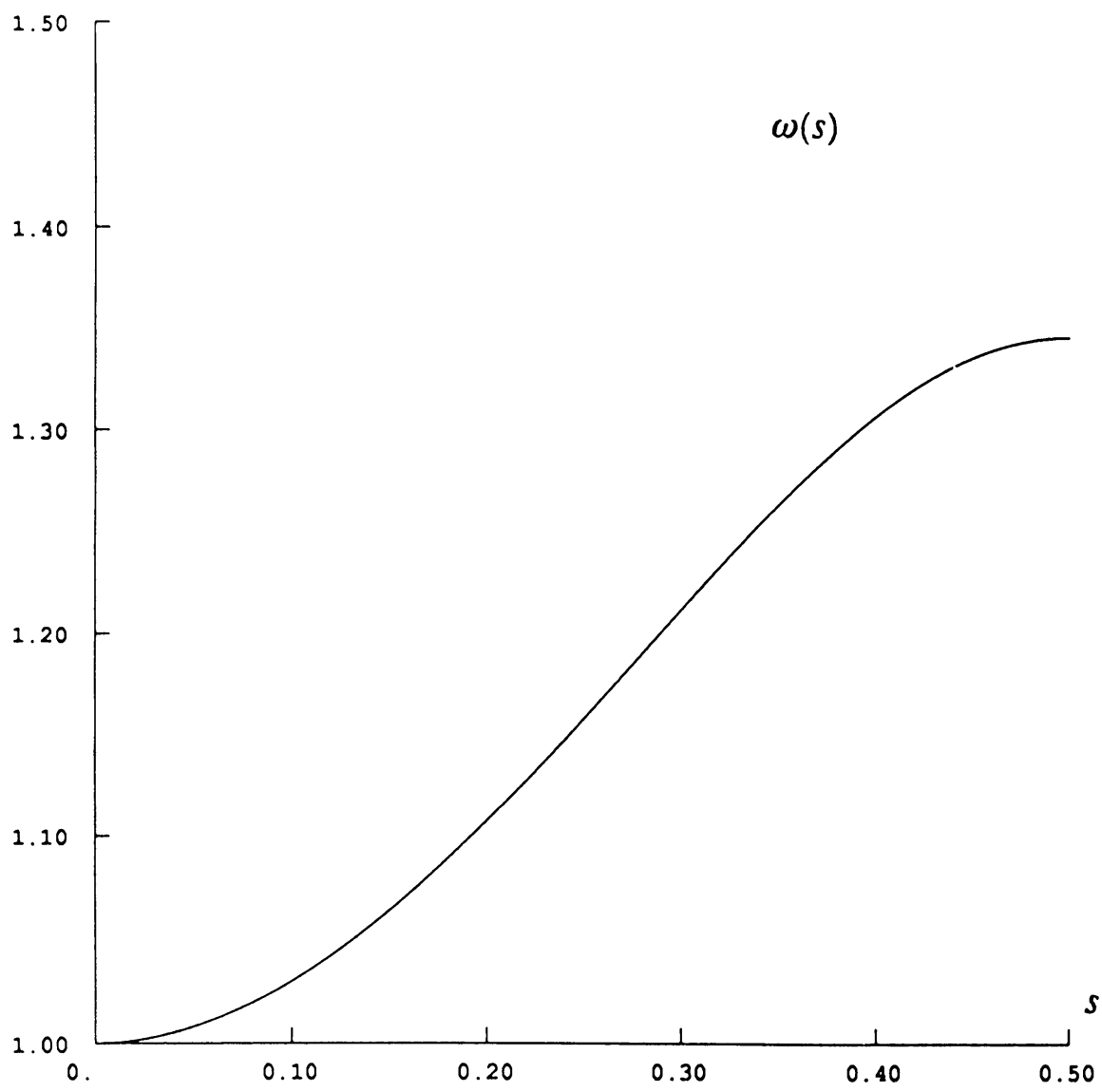

Figure 1. The function $\omega(s)$

Now for $s \in(0,1)$ let

$$
\omega(s)=\frac{\sum_{k=0}^{\infty}\left(1 /(k+s)^{2}+1 /(k+1-s)^{2}\right)}{\sum_{k=0}^{\infty}(-1)^{k}\left(1 /(k+s)^{2}+1 /(k+1-s)^{2}\right)} .
$$

Since $\omega(s)$ is symmetric about $\frac{1}{2}$, its maximum on the interval $(0,1)$ can be expressed as

$$
\omega=\sup _{s \in(0,1 / 2]} \omega(s) .
$$

As shown in Figure 1, $\omega(s)$ is a monotonic increasing function on the interval $\left(0, \frac{1}{2}\right]$. Thus $\omega=\omega\left(\frac{1}{2}\right) \approx 1.34688525$.

In the following it is shown that $\omega$ is an upper bound for $\left|B^{h}\right|_{0}$, and that this bound becomes increasingly sharp as $n \rightarrow \infty$.

Theorem 2. (i) When $n$ is sufficiently large, $\left|B^{h}\right|_{0} \leq \omega$;

(ii) $\lim _{n \rightarrow \infty}\left|B^{h}\right|_{0}=\omega$. 
Proof. For convenience we let

$$
a(m)= \begin{cases}1 /|m|, & m \in \mathbf{Z}^{*} \\ 1, & m=0\end{cases}
$$

Now

$$
\left|w_{h}\right|_{0}^{2}=\sum_{m \in \mathbf{Z}}\left|\hat{w}_{h}(m)\right|^{2}=\sum_{p \in \Lambda_{h}} \sum_{k \in \mathbf{Z}}\left|\hat{w}_{h}(k n+p)\right|^{2} .
$$

Since we have, by a simple calculation,

$$
\hat{w}_{h}(k n+p)(k n+p)=(-1)^{k} e^{-i k n \sigma_{1 / 2}} \hat{w}_{h}(p) p,
$$

it follows that

$$
\left|\hat{w}_{h}(k n+p)\right|^{2} a^{-2}(k n+p)=\left|\hat{w}_{h}(p)\right|^{2} a^{-2}(p) .
$$

Thus,

$$
\left|w_{h}\right|_{0}^{2}=\sum_{p \in \Lambda_{h}} \frac{\left|\hat{w}_{h}(p)\right|^{2}}{a^{2}(p)} \sum_{k \in \mathbf{Z}} a^{2}(k n+p) .
$$

Further, for $p \in \Lambda_{h}$ and with $w_{h}=B^{h} w$, we have

$$
\begin{aligned}
\hat{w}_{h}(p) & =\frac{1}{\sqrt{2 \pi}}\left(\left(Q^{h} A_{h}\right)^{-1} Q^{h} A w, P^{h} e^{i p s}\right) \\
& =\frac{1}{\sqrt{2 \pi}}\left(Q^{h} A w,\left(Q^{h} A_{h}\right)^{-1} P^{h} e^{i p s}\right) \quad(\text { see }(6)) \\
& =\frac{1}{\sqrt{2 \pi}}\left(Q^{h} A w, P^{h} e^{i p s}\right) \lambda_{p}^{-1},
\end{aligned}
$$

where $P^{h}$ is the orthogonal projection operator to $S^{h}(\Pi)$ under the inner product $(\cdot, \cdot)\left(\right.$ or $\left.(\cdot, \cdot)_{0}\right)$. In the last step above we have used Theorem 1 and the fact that

$$
P^{h} e^{i m s}=\alpha_{m} e_{h}^{m}(s) \text { for } m \in \mathbf{Z},
$$

with $\alpha_{m}=2(m h)^{-1} \sin (m h / 2) e^{i m \sigma_{1 / 2}}$. Another useful fact is

$$
Q^{h} e^{i m s}=\beta_{m} e_{h}^{m}(s) \text { for } m \in \mathbf{Z},
$$

with $\beta_{m}=e^{i m \sigma_{1 / 2}}$. Both of these facts will be used in the following argument without comment. Now

$$
\begin{aligned}
\left(Q^{h} A w, P^{h} e^{i p s}\right) & =\frac{1}{\sqrt{2 \pi}} \sum_{j \in \mathbf{Z}} \sum_{l \in \Lambda_{h}} a(j n+l) \hat{w}(j n+l)\left(Q^{h} e^{i(j n+l) s}, P^{h} e^{i p s}\right) \\
& =\frac{1}{\sqrt{2 \pi}} \sum_{j \in \mathbf{Z}} \sum_{l \in \Lambda_{h}} a(j n+l) \hat{w}(j n+l) \beta_{j n+l} \bar{\alpha}_{p}\left(e_{h}^{j n+l}(s), e_{h}^{p}(s)\right) \\
& =\sqrt{2 \pi} \bar{\alpha}_{p} \sum_{j \in \mathbf{Z}} \beta_{j n+p} a(j n+p) \hat{w}(j n+p) .
\end{aligned}
$$


Thus,

$$
\begin{aligned}
\left|\hat{w}_{h}(p)\right|^{2} & =\left|\lambda_{p}^{-1} \alpha_{p}\right|^{2}\left|\sum_{j \in \mathbf{Z}} \beta_{j n+p} a(j n+p) \hat{w}(j n+p)\right|^{2} \\
& \leq\left|\lambda_{p}^{-1} \alpha_{p}\right|^{2} \sum_{k \in \mathbf{Z}} a^{2}(k n+p) \sum_{j \in \mathbf{Z}}|\hat{w}(j n+p)|^{2},
\end{aligned}
$$

and therefore

$$
\left|w_{h}\right|_{0}^{2} \leq \sum_{p \in \Lambda_{h}} \sum_{j \in \mathbf{Z}}|\hat{w}(j n+p)|^{2}-\frac{\left|\lambda_{p}^{-1} \alpha_{p}\right|^{2}}{a^{2}(p)}\left(\sum_{k \in \mathbf{Z}} a^{2}(k n+p)\right)^{2} .
$$

However, by a direct calculation we find that

$$
\frac{\left|\lambda_{p}^{-1} \alpha_{p}\right|^{2}}{a^{2}(p)}\left(\sum_{k \in \mathbf{Z}} a^{2}(k n+p)\right)^{2}= \begin{cases}1+h^{2} / 12, & p=0, \\ \omega^{2}(p / n), & p \in \Lambda_{h}^{*} .\end{cases}
$$

Since $\omega(s)>1$ for $s \in\left(0, \frac{1}{2}\right]$, we therefore have, when $n$ is sufficiently large,

$$
\left|w_{h}\right|_{0}^{2} \leq \omega^{2} \sum_{p \in \Lambda_{h}} \sum_{j \in \mathbf{Z}}|\hat{w}(j n+p)|^{2}=\omega^{2}|w|_{0}^{2} .
$$

This completes the proof of (i).

In order to show that $\lim _{n \rightarrow \infty}\left|B^{h}\right|_{0}=\omega$, for each $0 \leq l \leq(n-1) / 2$ define a function $w^{l} \in H^{0}$ with the following Fourier coefficients: for $j \in \mathbf{Z}, p \in \Lambda_{h}$,

$$
\hat{w}^{\prime}(j n+p)= \begin{cases}\bar{\beta}_{j n \pm l} a(j n \pm 1), & p= \pm l, \\ 0, & \text { otherwise }\end{cases}
$$

and follow the same procedure as in the proof of (i) above. We then obtain

$$
\left|w_{h}^{l}\right|_{0}^{2}= \begin{cases}\left(1+h^{2} / 12\right)\left|w^{l}\right|_{0}^{2}, & l=0, \\ \omega^{2}(l / n)\left|w^{l}\right|_{0}^{2}, & 1 \leq l \leq(n-1) / 2 .\end{cases}
$$

This shows that in fact

$$
\left|B^{h}\right|_{0}=\max _{1 \leq l \leq(n-1) / 2}\left\{\omega\left(\frac{l}{n}\right), \sqrt{1+\frac{h^{2}}{12}}\right\}
$$

and hence $\lim _{n \rightarrow \infty}\left|B^{h}\right|_{0}=\omega$. The proof is now complete.

Theorem 2 tells us that $\omega$ can be viewed essentially as the value of $\left|B^{h}\right|_{0}$ when $n$ is sufficiently large.

It is clear that $\left(B^{h}\right)^{2}=B^{h}$, so $B^{h}$ is a projection operator onto $S^{h}(\Pi)$. Moreover, the projection operator $B^{h}$ has the following property, because $\left(I-B^{h}\right) w=\left(I-B^{h}\right)\left(w-P^{h} w\right)$, and $\left|B^{h}\right|_{0}$ is uniformly bounded. 
Proposition 1. We have $\left|\left(I-B^{h}\right) w\right|_{0} \leq c\left|\left(I-P^{h}\right) w\right|_{0}$, where $c$ is a constant independent of $n$.

This proposition shows that the collocation method for the circle is convergent, and the error is within a constant multiple of the best $L_{2}$ approximation. The first proof of this proposition was given in [13].

Next to the collocation method on the circle, we briefly consider the Galerkin method on the circle,

$$
P^{h} A w_{h}^{G}=P^{h} A w
$$

or equivalently,

$$
\left(A w_{h}^{G}, v_{h}\right)_{0}=\left(A w, v_{h}\right)_{0} \text { for } v_{h} \in S^{h}(\Pi) .
$$

Since this equation is equivalent to (see [20])

$$
\left(w_{h}^{G}, v_{h}\right)_{-1 / 2}=\left(w, v_{h}\right)_{-1 / 2} \text { for } v_{h} \in S^{h}(\Pi),
$$

$w_{h}^{G}$ is exactly the orthogonal projection of $w$ to $S^{h}(\Pi)$ under the particular inner product $(\cdot, \cdot)_{-1 / 2}$. To distinguish this particular orthogonal projection from $P^{h}$, we write $w_{h}^{G}=P_{-1 / 2}^{h} w$. Then (7) implies $\left|P_{-1 / 2}^{h}\right|_{-1 / 2}=1$, and we obtain that $P_{-1 / 2}^{h}=\left(P^{h} A_{h}\right)^{-1} P^{h} A$.

A similar explanation can be given for the collocation method on the circle. Indeed, let $S_{*}^{h}(\Pi):=\operatorname{span}\left\{\delta\left(s-\sigma_{j+1 / 2}\right): j=0,1, \ldots, n-1\right\}$, where $\delta(s)$ is the delta function. It is obvious that the interpolation projection $Q^{h} w$ of a function $w \in C(2 \pi)$ (or $\in H^{1 / 2+\varepsilon}$ with $\varepsilon>0$ ) onto $S^{h}(\Pi)$ is the unique solution of a Petrov-Galerkin approximation equation:

$$
\left(w_{h}, v_{h}\right)_{0}=\left(w, v_{h}\right)_{0} \quad \text { for } v_{h} \in S_{*}^{h}(\Pi) .
$$

Hence, the interpolation projection $Q^{h} w$ of $w$ to $S^{h}(\Pi)$ can be defined as the solution of equation (8). This definition holds for the particular inner product $(\cdot, \cdot)_{0}$, but it obviously can be extended to the more general inner product $(\cdot, \cdot)_{t}$, with $t$ a nonpositive real number.

Definition. For $t \leq 0$, and $w \in H^{2 t+1 / 2+\varepsilon}$ with $\varepsilon>0$, if $w_{h} \in S^{h}(\Pi)$ satisfies

$$
\left(w_{h}, v_{h}\right)_{t}=\left(w, v_{h}\right)_{t} \text { for } v_{h} \in S_{*}^{h}(\Pi),
$$

then $w_{h}$ is called the interpolation projection of $w$ to $S^{h}(\Pi)$ under the inner product $(\cdot, \cdot)_{t}$. It is denoted by $w_{h}=Q_{t}^{h} w$.

In this definition, the condition $t \leq 0$ is required so that the left-hand side of equation (9) makes sense. The assumption $w \in H^{2 t+1 / 2+\varepsilon}$ with $\varepsilon>0$ makes the right-hand side of (9) finite.

It is obvious that the collocation equation

$$
Q^{h} A w_{h}=Q^{h} A w
$$


is equivalent to

$$
\left(A w_{h}, v_{h}\right)_{0}=\left(A w, v_{h}\right)_{0} \text { for } v_{h} \in S_{*}^{h}(\Pi),
$$

and that this equation is equivalent to (see [20])

$$
\left(w_{h}, v_{h}\right)_{-1 / 2}=\left(w, v_{h}\right)_{-1 / 2} \text { for } v_{h} \in S_{*}^{h}(\Pi) .
$$

Thus the collocation solution $B^{h} w$ is the unique solution of equation (9) with $t=-\frac{1}{2}$. That is to say, $B^{h}$ is just the interpolation projection to $S^{h}(\Pi)$ under the particular inner product $(\cdot, \cdot)_{-1 / 2}$. Thus, we may write $B^{h}=Q_{-1 / 2}^{h}$.

\section{CONVERGENCE OF COLlOCATION ON THE POLYGON}

In this section we consider the convergence of the collocation method $(2)^{*}$ on a polygonal boundary. For this purpose we employ the results of the last section and show an equivalence of the collocation equation $(2)^{*}$ to a particular projection approximation of a second-kind Fredholm equation. We then prove the convergence of the collocation method on the polygonal boundary under a certain restriction on the angles of the corners.

As in the analysis on a polygonal boundary in [20], the operator $K$ in $(1)^{*}$ can be decomposed as

$$
K=A(I+L+E)
$$

with $I$ an identity, $E$ compact on $L_{2}(2 \pi)$, and $L$ bounded on $L_{2}(2 \pi)$ by $|L|_{0} \leq \max _{1 \leq r \leq r}\left\{1-\cos \left(\chi_{r} \pi / 2\right)\right\}$. The number $\chi_{r} \in(-1,1)$ is defined by requiring $\left(1-\chi_{r}\right) \pi$ to be the interior angle $\widehat{v_{r-1} v_{r} v_{r+1}}$ at the corner point $v_{r}$. The operator $K: H^{0} \rightarrow H^{1}$ is invertible when the transfinite diameter (or capacity) $C_{\Gamma}$ is not 1 . Because of this decomposition, the collocation equation $(2)^{*}$ can be rewritten as

$$
Q^{h} A w_{h}+Q^{h} A(L+E) w_{h}=Q^{h} \bar{f} .
$$

Let $\bar{f}=A f^{*}$. Then $(10)$ is equivalent to

$$
w_{h}+B^{h}(L+E) w_{h}=B^{h} f^{*} .
$$

This has the form of a projection method for a standard second-kind Fredholm equation (see $[1,12,4,6])$ with the particular projection operator $B^{h}$ onto $S^{h}(\Pi)$. Since $E$ is compact, $L$ is bounded by

$$
|L|_{0} \leq \max _{1 \leq r \leq i}\left\{1-\cos \left(\chi_{r} \pi / 2\right)\right\},
$$

and $B^{h}$ is bounded by $\left|B^{h}\right|_{0} \leq \omega$, the standard theory yields the following convergence theorem on the polygonal boundary.

Theorem 3. Assume $C_{\Gamma} \neq 1$. If

$$
\omega \max _{1 \leq r \leq ;}\left\{1-\cos \frac{\chi_{r} \pi}{2}\right\}<1
$$


when $n$ is sufficiently large, the collocation equation $(2)^{*}$ is uniquely solvable on $S^{h}(\Pi)$ for any continuous function $\bar{f}$. Furthermore, if $w \in L_{2}(2 \pi)$ is the solution of equation $(1)^{*}$, then

$$
\left|w_{h}-w\right|_{0} \leq c\left|\left(I-P^{h}\right) w\right|_{0},
$$

where $c$ is a constant independent of $n$.

Proof. Since $|L|_{0}<1$ and $\left|B^{h} L\right|_{0} \leq \omega|L|_{0}<1$, both $(I+L)^{-1}$ and $\left(I+B^{h} L\right)^{-1}$ exist, and

$$
\left|\left(I+B^{h} L\right)^{-1}\right|_{0} \leq \frac{1}{1-\omega|L|_{0}} .
$$

Now

$$
I+B^{h}(L+E)=\left(I+B^{h} L\right)\left(I+\left(I+B^{h} L\right)^{-1} B^{h} E\right),
$$

and

$$
I+\left(I+B^{h} L\right)^{-1} B^{h} E=I+(I+L)^{-1} E-\left(I+B^{h} L\right)^{-1}\left(I-B^{h}\right)(I+L)^{-1} E .
$$

Because $\left|\left(I-B^{h}\right) v\right|_{0} \rightarrow 0$ as $n \rightarrow \infty$, for $v \in L_{2}(2 \pi)$, and because $E$ is compact on $H^{0}$, so that $(I+L)^{-1} E$ is also compact, we have

$$
\left|\left(I+B^{h} L\right)^{-1}\left(I-B^{h}\right)(I+L)^{-1} E\right|_{0} \leq \frac{1}{1-\omega|L|_{0}}\left|\left(I-B^{h}\right)(I+L)^{-1} E\right|_{0} \rightarrow 0
$$

as $n \rightarrow \infty$. However, from the analysis in [20], the assumption $C_{\Gamma} \neq 1$ ensures that $(I+L+E)^{-1}$ exists, so that $\left(I+(I+L)^{-1} E\right)$ is invertible. Therefore, when $n$ is sufficiently large, there is a constant $c^{1}$ independent of $n$ such that

$$
\left|\left(I+\left(I+B^{h} L\right)^{-1} B^{h} E\right)^{-1}\right|_{0} \leq c^{1} .
$$

It follows from (12) that when $n$ is sufficiently large, equation $(10)^{*}$ is uniquely solvable, and so is the collocation equation $(2)^{*}$. Moreover,

$$
\left|\left(I+B^{h}(L+E)\right)^{-1}\right|_{0} \leq \frac{c^{1}}{1-\omega|L|_{0}} .
$$

Further, from $(I+L+E) w=f^{*}$ there follows

$$
w+B^{h}(L+E) w=B^{h} f^{*}+\left(I-B^{h}\right) w .
$$

Thus,

$$
\left(I+B^{h}(L+E)\right)\left(w-w_{h}\right)=\left(I-B^{h}\right) w,
$$

and because of (13),

$$
\left|w-w_{h}\right|_{0} \leq \frac{c^{1}}{1-\omega|L|_{0}}\left|\left(I-B^{h}\right) w\right|_{0} .
$$

Applying Proposition 1, we obtain

$$
\left|w-w_{h}\right|_{0} \leq c\left|\left(I-P^{h}\right) w\right|_{0},
$$

with $c$ independent of $n$. The proof is now complete. 
From the proof above we find that the restriction (11) on the angles at the corners derives from the estimate $\left|B^{h} L\right|_{0} \leq\left|B^{h}\right|_{0}|L|_{0}$, and $\left|B^{h}\right|_{0} \approx \omega$. The restriction may not be necessary, since $\left|B^{h} L\right|_{0}$ could be less than 1 even if (11) is not satisfied. However, because the operators $B^{h}$ and $L$ are quite complicated, a direct estimate of $\left|B^{h} L\right|_{0}$ is difficult. A similar restriction occurs for the second-kind boundary integral equation methods (see for example [5]). As shown in [5], the restriction may be more severe than necessary, or may not be needed at all.

The restriction (11) is equivalent to

$$
\max _{1 \leq r \leq i}\left|\chi_{r}\right|<4 \pi^{-1} \arcsin (2 \omega)^{-1 / 2} \approx 0.83417205 \text {. }
$$

From this, we see more clearly that convergence holds when each of the interior angles of the polygon is within the interval $\left(29.84903087^{\circ}, 330.15096913^{\circ}\right)$.

\section{Superconvergence}

In practice, one is usually more interested in quantities such as the 'flux' through the boundary $\Gamma$,

$$
\int_{\Gamma} g(y) d y=\int_{-\pi}^{\pi} w(\sigma) d \sigma=(w, 1),
$$

and the potential at $\tau \notin \Gamma$,

$$
-\int_{\Gamma} \log |\tau-y| g(y) d y=-\int_{-\pi}^{\pi} \log |\tau-v(\sigma)| w(\sigma) d \sigma=\left(k_{\tau}, w\right),
$$

rather than in the value of $w$. That is to say, one is often more interested in the linear functional

$$
\int_{-\pi}^{\pi} w(\sigma) v(\sigma) d \sigma=(w, v)
$$

where $v$ is well behaved. The error in the approximation of $(w, v)$ by $\left(w_{h}, v\right)$ is given by

Theorem 4. Under the conditions of Theorem 3,

$$
\left|(w, v)-\left(w_{h}, v\right)\right| \leq c h\left|w-w_{h}\right|_{0}|v|_{1} \quad \text { for } v \in H^{1}
$$

where $c$ is a constant independent of $n$.

Proof. Since $K: H^{0} \rightarrow H^{1}$ is invertible, for $v \in H^{1}$ there exists a $u \in H^{0}$ such that $K u=v$ and

$$
|u|_{0}=\left|K^{-1} v\right|_{0} \leq c|v|_{1}
$$

with $c$ a constant. Thus,

$$
\begin{aligned}
(w, v)-\left(w_{h}, v\right) & =\left(w-w_{h}, K u\right)=\left(K\left(w-w_{h}\right), u\right) \\
& =\left(\left(I-Q^{h}\right) K\left(w-w_{h}\right), u\right),
\end{aligned}
$$

and so

$$
\left|(w, v)-\left(w_{h}, v\right)\right| \leq\left|\left(I-Q^{h}\right) K\right|_{0}\left|w-w_{h}\right|_{0} c|v|_{1} \quad \text { for } w, v \in H^{2} .
$$


However, it is well known that there is a constant $c$ independent of $n$ such that

$$
\left|\left(I-Q^{h}\right) v\right|_{0} \leq c h|v|_{1}
$$

Therefore,

$$
\left|\left(I-Q^{h}\right) K\right|_{0} \leq c h,
$$

because $K: H^{0} \rightarrow H^{1}$ is bounded. Thus the theorem follows.

Set $w \in H^{1}$. From Theorem 4 we obtain

$$
\left|(w, v)-\left(w_{h}, v\right)\right| \leq c h^{2}|w|_{1}|v|_{1} .
$$

While Saranen [14] has shown that the optimal order of convergence on a sufficiently smooth boundary is

$$
\left|(w, v)-\left(w_{h}, v\right)\right| \leq c h^{3}|w|_{2}|v|_{2},
$$

the assumption in that paper is: the operator $K-A: H^{0} \rightarrow H^{2}$ is at least bounded. This assumption fails in the case of polygons. The reason is that even when the function $\bar{f}(s)$ on the right-hand side is well behaved, the solution $w(s)$ is still singular at the corner points. That is to say, there exists a $w \in H^{0}$ and $\notin H^{1}$ such that $K w$ is a smooth function, and then $(K-A) w=K w-A w \in H^{1}$ and $\notin H^{2}$.

Applications. It is known that near the corner $v_{j}$ the function $g(v(s))$ can generally be expected to have a singularity of the form $\left|s-s_{j}\right|^{\beta_{j}}$, where $\beta_{j}$ usually has the value $-\left|\chi_{j}\right| /\left(1+\left|\chi_{j}\right|\right)>-0.5$, but could be larger in some situations (see [19]). Using the results of Graham [11], we obtain

$$
\left|P^{h} g(v(s))-g(v(s))\right|_{0}=O\left(h^{\beta+1 / 2}\right),
$$

where $\beta=\min _{1 \leq j \leq i} \beta_{j}$.

(1) Rate of convergence for the capacity $C_{\Gamma}$. Here we calculate the quantity

$$
C_{\Gamma}=\exp \left(-\left(\int_{\Gamma} \lambda_{\Gamma}\right)^{-1}\right)=\exp \left(-\left(\int_{-\pi}^{\pi} w_{\lambda}(\sigma) d \sigma\right)^{-1}\right)
$$

by the approximation $C_{\Gamma}^{h}=\exp \left(-\left(\int_{-\pi}^{\pi} w_{\lambda}^{h}(\sigma) d \sigma\right)^{-1}\right)$, where $\lambda_{\Gamma}$ is the solution of equation (1) with the right-hand side $f=1, w_{\lambda}$ is the solution of equation $(1)^{*}$ with the right-hand side $\bar{f}=\frac{1}{\pi}$, and $w_{\lambda}^{h}$ is the solution of the corresponding collocation equation $(2)^{*}$. Theorem 4 and (14) give

$$
\left|\int_{-\pi}^{\pi} w_{\lambda}-\int_{-\pi}^{\pi} w_{\lambda}^{h}\right|=\left|\left(w_{\lambda}-w_{\lambda}^{h}, 1\right)\right| \leq c h\left|w_{\lambda}-w_{\lambda}^{h}\right|_{0}=O\left(h^{\beta+3 / 2}\right) .
$$

Therefore,

$$
C_{\Gamma}-C_{\Gamma}^{h}=O\left(h^{\beta+3 / 2}\right)
$$


(2) Rate of convergence for the potential. Here we approximate the exact potential at $\tau \notin \Gamma$,

$$
u(\tau)=-\int_{\Gamma} \log |\tau-y| g(y) d y=-\int_{-\pi}^{\pi} \log |\tau-v(\sigma)| w(\sigma) d \sigma,
$$

by

$$
u^{h}(\tau)=-\int_{-\pi}^{\pi} \log |\tau-v(\sigma)| w^{h}(\sigma) d \sigma
$$

where $w^{h}$ is the solution of equation $(2)^{*}$. Since $k_{\tau}(s)=-\log |\tau-v(s)| \in H^{1}$, Theorem 4 and (14) give

$$
\left|u(\tau)-u^{h}(\tau)\right|=\left|\left(k_{\tau}, w-w^{h}\right)\right| \leq c h\left|w-w^{h}\right|_{0}=O\left(h^{\beta+3 / 2}\right) .
$$

The estimate $O\left(h^{\beta+3 / 2}\right)$ for the rate of convergence of the capacity and the potential may be capable of improvement. A numerical experiment shows that the practical rate is more like the one for the Galerkin method, i.e., $O\left(h^{2(\beta+1)}\right)$ rather than $O\left(h^{\beta+3 / 2}\right)$. However, a theoretical analysis yielding this order is not yet known.

\section{ACKNOWLEDGMENT}

The author is grateful to Professor Ian Sloan for valuable suggestions.

\section{REFERENCES}

1. P. M. Anselone, Collectively compact operator approximation theory and applications to integral equations, Prentice-Hall, Englewood Cliffs, N.J., 1971.

2. D. N. Arnold and W. L. Wendland, On the asymptotic convergence of collocation methods, Math. Comp. 41 (1983), 349-381.

3. Math. 47 (1985), 317-341.

4. K. E. Atkinson, A survey of numerical methods for the solution of Fredholm integral equations of the second kind, SIAM, Philadelphia, Pa., 1976.

5. K. E. Atkinson and F. R. de Hoog, Collocation methods for a boundary integral equation on a wedge, in Treatment of Integral Equations by Numerical Methods (C. T. H. Baker and G. F. Miller, eds.), Academic Press, New York, 1983.

6. C. T. H. Baker, The numerical treatment of integral equations, Oxford Univ. Press, 1977.

7. S. Christiansen, On two methods for elimination of non-unique solutions of an integral equation with logarithmic kernel, Applicable Anal. 13 (1982), 1-18.

8. M. Costabel and E. P. Stephan, Collocation methods for integral equations on polygons, in Innovative Numerical Methods in Engineering (R. P. Shaw et al., eds.), Proc. 4th Internat. Sympos., Georgia Tech., Atlanta, GA, Springer-Verlag, Berlin and New York, 1986, pp. 43-50.

9. Math. Comp. 49 (1987), 461-478.

10. F. R. de Hoog, Product integration techniques for the numerical solution of integral equations, Ph.D. Thesis, Australian National University, 1973.

11. I. G. Graham, Estimates for the modulus of smoothness, J. Approx. Theory 44 (1985), 95-112.

12. M. A. Krasnoselskii et al., Approximate solution of operator equations, Noordhoff, Groningen, 1972. 
13. J. Saranen and W. L. Wendland, On the asymptotic convergence of collocation methods with spline functions of even degree, Math. Comp. 171 (1985), 91-108.

14. J. Saranen, The convergence of even degree spline collocation solution for potential problems in smooth domains of the plane, Numer. Math. 53 (1988), 499-512.

15. G. Schmidt, On spline collocation methods for boundary integral equations in the plane, Math. Appl. Sci. 7 (1985), 74-89.

16. __ On E-collocation for pseudodifferential equations on a closed curve, Math. Nachr. 126 (1986), 183-196.

17. I. Sloan and A. Spence, The Galerkin method for integral equations of the first kind with logarithmic kernel: Theory, IMA J. Numer. Anal. 8 (1988), 105-122.

18. _ The Galerkin method for integral equations of the first kind with logarithmic kernel: Applications, IMA J. Numer. Anal. 8 (1988), 123-140.

19. Y. Yan and I. Sloan, Mesh grading for integral equations of the first kind with logarithmic kernel, SIAM J. Numer. Anal. 26 (1989), 574-587.

20. __ On integral equations of the first kind with logarithmic kernel, J. Integral Equations Appl. 1 (1988), No. 4, 1-31.

Center for Computational Sciences, University of Kentucky, Lexington, Ky 40506. E-mail: yan@ukcc.uky.edu 